\title{
optica
}

\section{Quantitative interior x-ray nanotomography by a hybrid imaging technique: supplementary material}

\author{
Manuel Guizar-Sicairos ${ }^{1, *}$, JaAP J. Boon ${ }^{2}$, Kevin Mader $^{1,3}$, Ana Diaz ${ }^{1}$, \\ Andreas Menzel ${ }^{1}$, And Oliver BunK ${ }^{1}$ \\ ${ }^{1}$ Paul Scherrer Institut, 5232 Villigen PSI, Switzerland \\ ${ }^{2}$ FOM Institute AMOLF, 1098XG Amsterdam, The Netherlands \\ ${ }^{3}$ Institute for Biomedical Engineering, Swiss Federal Institute of Technology and University of Zurich, 8093 Zürich, Switzerland \\ *Corresponding author: manuel.guizar-sicairos@psi.ch
}

Published 19 March 2015

This document provides supplementary information to "Quantitative interior x-ray nanotomography by a hybrid imaging technique," http://dx.doi.org/10.1364/optica.2.000259.

(C) 2015 Optical Society of America

http://dx.doi.org/10.1364/optica.2.000259.s001

\section{X-RAY MEASUREMENTS}

X-ray measurements were performed at the cSAXS beamline, Swiss Light Source, Paul Scherrer Institut, using monochromatic radiation of $6.2 \mathrm{keV}$ photon energy defined by a double crystal Si (111) monochromator. A 3D piezoelectric scanning system was mounted on coarse positioning motors and a rotation stage, an experimental configuration described in Ref. [1]. This system allows for scanning the sample transverse to the direction of X-ray propagation for sample orientations that span the full angular range from $0^{\circ}$ to $360^{\circ}$. Diffraction patterns were measured using a Pilatus photon-counting detector [2] with a pixel size of $172 \mu \mathrm{m}$ located 7.24 meters from the sample.

\section{BEAM DEFLECTION TOMOGRAPHY}

The beam incident on the sample was defined by a $\sim 1 \mu \mathrm{m}$ pinhole. During acquisition the piezo fast axis was set to a constant speed [3] while X-ray diffraction patterns were measured on the fly with 0.04 second exposure time and 0.003 second readout time such that the spacing between subsequently measured points was $1 \mu \mathrm{m}$. The angular deflection of the X-ray beam was obtained using the distance between sample and detector by computing the transverse displacement of the diffraction pattern with respect to the pattern obtained without a sample. Such displacement was accurately and efficiently computed to $1 / 20$ of a pixel using selective upsampling cross-correlation [4], which proved more accurate than a center-of-mass analysis [3]. Transverse scans were performed at 60 equally spaced sample orientations, covering an angular range of $180^{\circ}$, with a field of view (FOV) of $84 \times 120 \mu \mathrm{m}^{2}$.

The angular deviation of the beam $\alpha_{x}$ and $\alpha_{y}$ are related to the gradient of the projected phase, $\Phi(x, y)$, by

$$
\frac{\partial \Phi(x, y)}{\partial x}=\frac{2 \pi}{\lambda} \sin \alpha_{x}(x, y)
$$

where $(x, y)$ are the Cartesian coordinates transverse to the direction of X-ray propagation $z, \lambda$ is the photon wavelength, and a similar expression exists for $\alpha_{y}$. Using a modified filtered back projection algorithm (FBP) the 3D distribution of the real part of the refractive index, $\delta(x, y, z)$, can be quantitatively reconstructed from measurements of the phase gradient at different sample orientations [5, 6]. Finally, for photon energies away from absorption edges of the atomic species in the sample the electron density can be obtained by

$$
n_{e}(x, y, z)=\frac{2 \pi \delta(x, y, z)}{\lambda^{2} r_{0}}
$$

where $r_{0}$ denotes the classical electron radius.

\section{ALIGNMENT OF HIGH-RESOLUTION PROJECTIONS USING TOMOGRAPHIC CONSISTENCY}

High-resolution interior projections were measured using ptychography [1,7-9] with a FOV of $32 \times 30 \mu \mathrm{m}$ at 450 equally spaced angular orientations that covered a range of $180^{\circ}$. The object complex-valued transmissivity for each orientation was reconstructed to a pixel size of $43.8 \mathrm{~nm}$ using the difference map algorithm [9] followed by maximum likelihood refinement $[8,10]$. From the complex-valued reconstructions phase projections of the sample were obtained and unwrapped [11].

Because of thermal drifts during the data acquisition the phase projections needed to be aligned prior to tomographic 
reconstruction. For alignment in the vertical direction we used the method in Ref. [12], while for alignment in the horizontal direction we developed an iterative sinogram alignment technique based on tomographic consistency, as detailed in the main manuscript. Figure S1(A) shows an axial tomographic slice computed using the FBP before alignment of projections where significant noise and artifacts are observed. After postprocessing alignment a significant improvement in image quality is evident, as shown in Figure S1(B).

\section{QUANTITATIVE INTERIOR TOMOGRAPHY}

An initial estimate of the position of the interior region of interest within the overview tomogram was readily taken from the readout position values of the stages and was later refined using non-linear optimization. A section from the overview tomogram was upsampled using trilinear interpolation to the pixel size of the high-resolution interior projections and was then given as initial estimate to a conjugate gradient optimization algorithm to iteratively minimize Eq. (1) of the main manuscript by refining high-resolution details of the tomogram.

During the optimization, regions outside the internal FOV are also refined, this is sometimes referred to as "exterior tomography". Because the exterior region has effectively an incomplete angular coverage, the reconstructions outside the interior ROI have non-isotropic resolution, and artifacts are expected. To exemplify this, Figures S2(B) and S2(C) show coronal sections of the eggshell sample through the center and at the edge of the interior ROI, respectively. Although the section in Figure S2(C) is completely outside the interior ROI high-resolution features can be observed.

The noise level within the interior ROI was estimated to be $\sigma_{\mathrm{e}}=0.05 \mathrm{e} / \AA^{3}$ from the standard deviation of values within the empty cavity shown in Figure $2 \mathrm{~F}$ in the main text. The density fluctuations that can be observed in the more homogeneous regions between vesicles are within the noise level with a standard deviation $\sigma_{\mathrm{e}}=0.06 \mathrm{e} / \AA^{3}$.

\section{ANALYSIS OF LAYERED ARRANGEMENT OF VESI- CLES}

Identification and classification of the vesicles into layers was performed using k-means cluster analysis [13]. In contrast to standard analysis that defines the cluster by coordinates that denote the position of a point, here to identify layers of vesicles we define each layer cluster $S_{i}$ by a unit vector and spatial distance $\left(\hat{s}_{i}, d_{i}\right)$ such that $\hat{s}_{i} \cdot \hat{r}+d_{i}=0$ uniquely defines a plane in three-dimensional space. The within-cluster distance to minimize is the Euclidean distance from the centroid of the vesicle to the plane plus a penalty term based on individual vesicle orientation with respect to the principal orientation of vesicles within the cluster [14]. Since planes corresponding to different layers can intersect each other within the measured volume the latter was introduced to help disambiguate cases where a vesicle lies close to two planes and to favor grouping of vesicles with similar orientations. The algorithm was implemented using the FlexClust [15, 16] (v1.3-3) package in R (v2.15.2) [17].

\section{REFERENCES}

1. M. Dierolf, A. Menzel, P. Thibault, P. Schneider, C. M. Kewish, R. Wepf, O. Bunk, and F. Pfeiffer, "Ptychographic $\mathrm{x}$-ray computed tomography at the nanoscale," Nature 467, 436-439 (2010).
2. B. Henrich, A. Bergamaschi, C. Brönnimann, R. Dinapoli, E. F. Eikenberry, I. Johnson, M. Kobas, P. Kraft, A. Mozzanica, and B. Schmitt, "PILATUS: A single photon counting pixel detector for X-ray applications," Nucl. Instrum. Methods Phys. Res. A 607, 247-249 (2009).

3. A. Menzel, C. M. Kewish, P. Kraft, B. Henrich, K. Jefimovs, J. Vila-Comamala, C. David, M. Dierolf, P. Thibault, F. Pfeiffer, and O. Bunk, "Scanning transmission X-ray microscopy with a fast framing pixel detector," Ultramicroscopy 110, 1143-1147 (2010).

4. M. Guizar-Sicairos, S. T. Thurman, and J. R. Fienup, “Efficient subpixel image registration algorithms," Opt. Lett. 33, 156-158 (2008).

5. G. W. Faris and R. L. Byer, "Three-dimensional beamdeflection optical tomography of a supersonic jet," Appl. Opt. 27, 5202-5212 (1988).

6. F. Pfeiffer, C. Kottler, O. Bunk, and C. David, "Hard x-ray phase tomography with low-brilliance sources," Phys. Rev. Lett. 98, 108105 (2007).

7. H. M. L. Faulkner and J. M. Rodenburg, "Movable aperture lensless transmission microscopy: a novel phase retrieval algorithm," Phys. Rev. Lett. 93, 023903 (2004).

8. M. Guizar-Sicairos and J. R. Fienup, "Phase retrieval with transverse translation diversity: a nonlinear optimization approach," Opt. Express 16, 7264-7278 (2008).

9. P. Thibault, M. Dierolf, A. Menzel, O. Bunk, C. David, and F. Pfeiffer, "High-resolution scanning x-ray diffraction microscopy," Science 321, 379-382 (2008).

10. P. Thibault and M. Guizar-Sicairos, "Maximum-likelihood refinement for coherent diffractive imaging," New J. Phys. 14, 063004 (2012).

11. R. M. Goldstein, H. A. Zebker, and C. L. Werner, "Satellite radar interferometry: Two-dimensional phase unwrapping," Radio Science 23, 713-720 (1988).

12. M. Guizar-Sicairos, A. Diaz, M. Holler, M. S. Lucas, A. Menzel, R. A. Wepf, and O. Bunk, "Phase tomography from xray coherent diffractive imaging projections," Opt. Express 19, 21345-21357 (2011).

13. F. Leisch, "A toolbox for k-centroids cluster analysis," Computational Statistics \& Data Analysis 51, 526-544 (2006).

14. F. Graner, B. Dollet, C. Raufaste, and P. Marmottant,."Discrete rearranging disordered patterns, part I: Robust statistical tools in two or three dimensions," Eur. Phys. J. E 25, 349-369 (2008).

15. F. Leisch, "Neighborhood graphs, stripes and shadow plots for cluster visualization," Stat. Comput. 20, 457-469 (2010).

16. F. Leisch and B. Gruen, "Extending standard cluster algorithms to allow for group constraints," A. Rizzi, M. Vichi (Eds.), Compstat 2006-Proceedings in Computational Statistics, Physica Verlag, Heidelberg, Germany, 885-892 (2006).

17. $\mathrm{R}$ Development Core Team, "R: A language and environment for statistical computing," R Foundation for Statistical Computing, Vienna, Austria. ISBN 3-900051-07-0, (2011). 

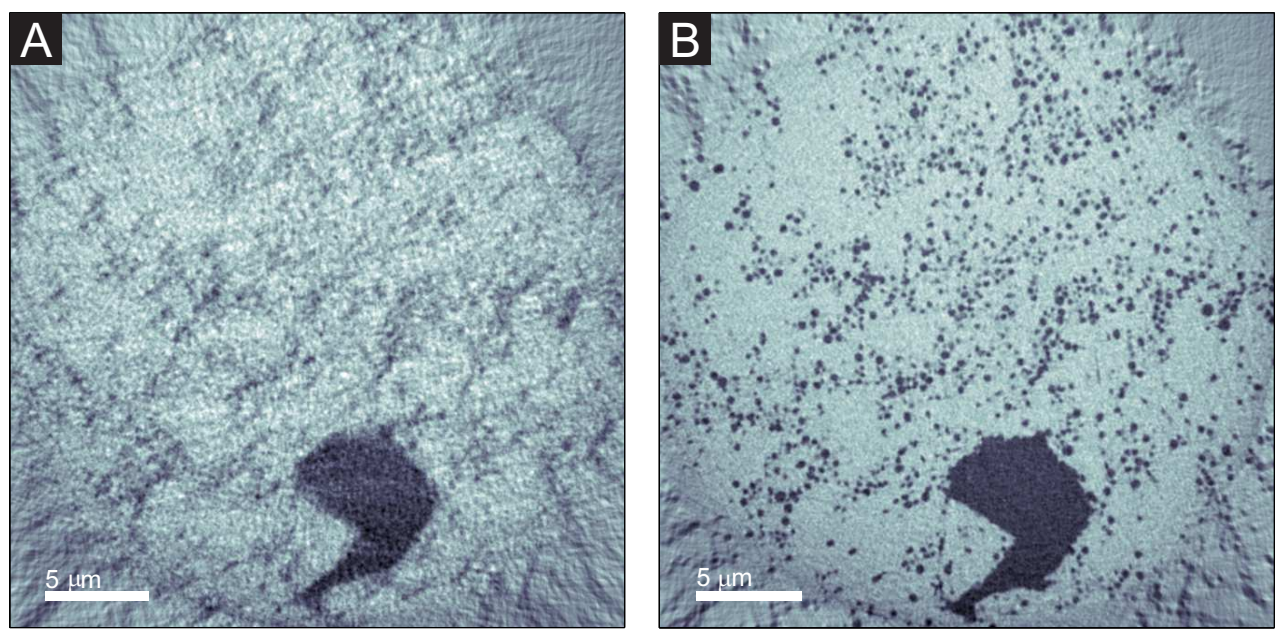

Fig. S1. Axial section reconstruction using FBP (A) before and (B) after sinogram alignment using tomographic consistency.
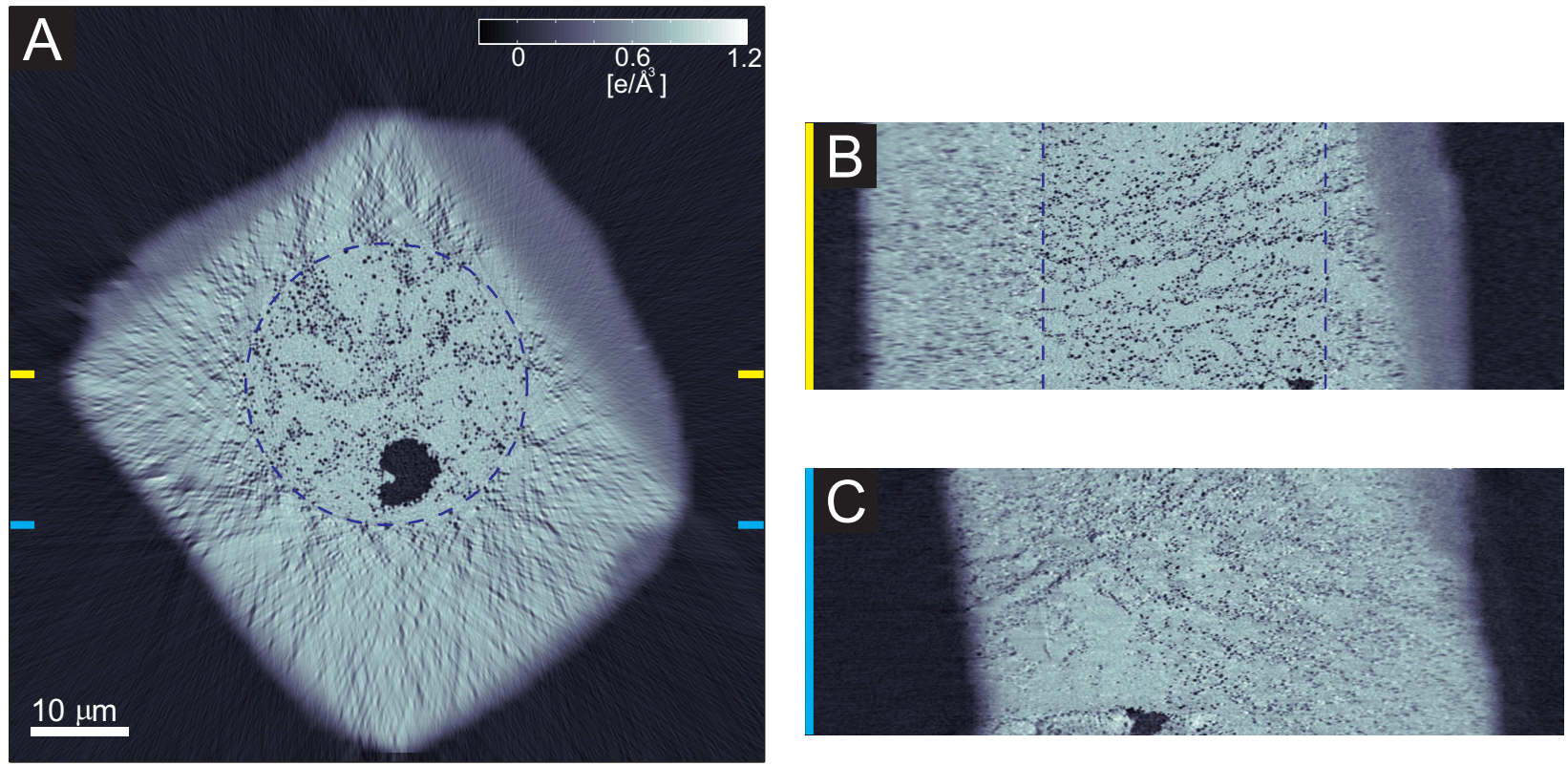

Fig. S2. (A) Axial section of hybrid tomographic reconstruction. Coronal sections through (B) the middle of the interior ROI and (C) outside the interior ROI, the coronal planes are indicated in (A) with yellow and cyan lines respectively. The limits of the interior $\mathrm{ROI}$ are indicated by blue dashed lines in (A) and (B). 